\title{
Distinct epigenetic regulation of tumor suppressor genes in putative cancer stem cells of solid tumors
}

\author{
HIROYUKI YASUDA $^{1}$, KENZO SOEJIMA ${ }^{1}$, HIDEO WATANABE ${ }^{3}$, ICHIRO KAWADA ${ }^{1}$, \\ ICHIRO NAKACHI ${ }^{1}$, SATOSHI YODA ${ }^{1}$, SOHEI NAKAYAMA ${ }^{1}$, RYOSUKE SATOMI ${ }^{1}$, \\ SHINNOSUKE IKEMURA ${ }^{1}$, HIDEKI TERAI ${ }^{1}$, TAKASHI SATO ${ }^{1}$, SADAFUMI SUZUKI ${ }^{2}$, \\ YUMI MATSUZAKI ${ }^{2}$, KATSUHIKO NAOKI ${ }^{1}$ and AKITOSHI ISHIZAKA ${ }^{1}$ \\ Departments of ${ }^{1}$ Pulmonary Medicine and ${ }^{2}$ Physiology, School of Medicine, Keio University, \\ 35 Shinanomachi, Shinjuku-ku, Tokyo 160-8582, Japan; ${ }^{3}$ Department of Medical Oncology, \\ Dana-Farber Cancer Institute, 44 Binney Street, Boston, MA 02115, USA
}

Received July 22, 2010; Accepted September 20, 2010

DOI: $10.3892 /$ ijo_00000807

\begin{abstract}
Epigenetic gene regulation plays essential roles in differentiation of embryonic and tissue stem cells. In these benign undifferentiated cells, some polycomb targeted genes are kept in a state of DNA hypomethylation and they have a distinct chromatin signature termed bivalent chromatin structure to maintain their plasticity. We hypothesized that cancer stem cells (CSC), the malignant counterpart of these cells, are also under the control of epigenetics like benign stem cells. We compared the DNA methylation and chromatin structure in 10 tumor suppressor genes between CSC and differentiated cancer cells of MCF7 and Huh7 cells. We found that the level of DNA methylation was indeed significantly lower in CSC, while surprisingly, the bivalent chromatin structure was more ubiquitously seen in differentiated cancer cells compared to CSC. However, repressive marks of chromatin structure, namely $\mathrm{H} 3 \mathrm{~K} 27 \mathrm{me} 3$ and EZH2, were significantly lower in CSC. As a consequence, CSC remained in a higher transcriptionally active chromatin state compared to differentiated cancer cells. We found that the differentiation of CSCs is also epigenetically regulated. These findings could help towards a comprehensive understanding of CSC, and also improve the development of eradicative therapies against human malignancies.
\end{abstract}

\section{Introduction}

Epigenetic gene regulation is an essential part of key biological processes such as differentiation, imprinting, and oncogenesis (1-5). This regulation includes chromatin modification or DNA

Correspondence to: Dr Kenzo Soejima, Department of Pulmonary Medicine, School of Medicine, Keio University, 35 Shinanomachi, Shinjuku-ku, Tokyo 160-8582, Japan

E-mail: ksoejima@cpnet.med.keio.ac.jp

Key words: epigenetics, cancer stem cells, DNA methylation methylation and controls the expression levels of various genes (6-8). Epigenetic regulation is particularly important in embryonic stem (ES) cells and tissue stem cells. DNA methylation, which is one of the most common types of epigenetic regulation, induces transcriptional silencing of genes $(9,10)$. DNA methylation has been reported to be related to the differentiation of ES cells and embryonal carcinoma cells (11-13). In cancer cells, aberrant DNA hypermethylation of tumor suppressor genes leads to silencing of these genes, which contribute to oncogenesis (1-4).

Chromatin modification includes the methylation, acetylation, or phosphorylation of histones (14). Recently, a distinct stem cell specific chromatin structure, a so-called 'bivalent' chromatin structure, has been reported (15). Normally, chromatins are either in a transcriptionally active or repressive state. Each state has state specific chromatin modifications, such as the trimethylation of lysine 27 of histone H3 in transcriptionally repressive chromatin, or the dimethylation or trimethylation of lysine 4 of histone $\mathrm{H} 3$ in transcriptionally active chromatin. However, in the bivalent chromatin state, these active and repressive chromatin marks coexist. The bivalent chromatin structure marks key developmental genes in ES cells. It has also been reported that Polycomb repressive complex (PRC), especially PRC2, plays critical roles in the formation of this unique chromatin pattern and the regulation of PRC targeted genes (16-18). It has been reported that some tumor suppressor genes are also the target of PRC and regulated epigenetically. In undifferentiated cells like ES cells and embryonal cancer cells, such tumor suppressor genes are marked by bivalent chromatin structure and lack aberrant DNA hypermethylation, although relatively differentiated adult cancer cells have aberrant DNA hypermethylation (19).

On the other hand, several previous reports have indicated that only rare cells in certain solid tumors, such as brain, breast, colon and liver cancers, as well as in hematological malignancies, have the capacity to initiate tumors (20-26). These rare cells are thought to be 'cancer stem cells (CSC)'. These cells are thought to have many properties common to normal stem cells, but their exact origins remain controversial. 
Table I. The primers used for MSP in the study.

\begin{tabular}{ll}
\hline Gene name & Sequence (5'-3')
\end{tabular}

APC

Methyl sense

Methyl antisense

Unmethyl sense

Unmethyl antisense

BRCA1

Methyl sense

Methyl antisense

Unmethyl sense

Unmethyl antisense

CDH1

Methyl sense

Methyl antisense

Unmethyl sense

Unmethyl antisense

CDKN2A
TATTGCGGAGTGCGGGTC

TCGACGAACTCCCGACGA

GTGTTTTATTGTGGAGTGTGGGTT

CCAATCAACAAACTCCCAACAA

TCGTGGTAACGGAAAAGCGC AAATCTCAACGAACTCACGCCG TTGGTTTTTGTGGTAATGGAAAAGTGT CAAAAAATCTCAACAAACTCACACCA

TGTAGTTACGTATTTATTTTTAGTGGCGTC CGAATACGATCGAATCGAACCG TGGTTGTAGTTATGTATTTATTTTTAGTGGTGTT ACACCAAATACAATCAAATCAAACCAAA

TTATTAGAGGGTGGGGCGGATCGC GACCCCGAACCGCGACCGTAA TTATTAGAGGGTGGGGTGGATTGT CAACCCCAAACCACAACCATAA

GGATAGTCGGATCGAGTTAACGTC CCCTCCCAAACGCCGA GGAGGATAGTTGGATTGAGTTAATGTT CAAATCCCTCCCAAACACCAA

ACGAGTTTAACGTCGCGGTC ACCCCCCAAACCGTTAAAAC TGTTGTTTATGAGTTTAATGTTGTGGTT AAAAAAACCCCCCAAACCATT

GCGGGTTTGGGTTTTTACGC CGACGCCGACCCCACTAAA GGGTGTGGGTTTGGGTTTTTATGT CCATAAACAACACCAACCCCACTAAA

TTTCGACGTTCGTAGGTTTTCGC GCACTCTTCCGAAAACGAAACG TTTGTGTTTTGATGTTTGTAGGTTTTTGT AACTCCACACTCTTCCAAAAACAAAACA

TCGAGAACGCGAGCGATTCG GACCAATCCAACCGAAACGA TTGAGAATGTGAGTGATTTGA AACCAATCCAACCAAAACAA

GGGTTCGTTTTGTGGTTTCGTTC TAACCCGATTAAACCCGTACTTCG GGGGTTTGTTTTGTGGTTTTGTTT AACATAACCCAATTAAACCCATACTTCA 
Table I. Continued.

Gene name $\quad$ Sequence (5'-3')

SFRP1

Methyl sense

Methyl antisense

Unmethyl sense

Unmethyl antisense

TIMP3

Methyl sense

Methyl antisense

Unmethyl sense

Unmethyl antisense

\author{
TGTAGTTTTCGGAGTTAGTGTCGCGC \\ CCTACGATCGAAAACGACGCGAACG \\ GTTTTGTAGTTTTTGGAGTTAGTGTTGTGT \\ CTCAACCTACAATCAAAAACAACACAAACA
}

CSCs are thought to play essential roles in cancer biology, including tumor initiation, progression and tumor relapse after chemotherapy. To eradicate cancer cells, CSC targeted chemotherapy is anticipated. For this purpose, further understanding of characteristics of CSC is mandatory. To understand the biology of stem cells, elucidation of epigenetics is indispensable. It has been proposed that epigenetic gene regulation is also essential not only in benign stem cells but also in CSC. However, no report has directly demonstrated any evidence that supports this proposal so far.

We hypothesized that the epigenetic regulation of benign stem cells can be also applied to CSC. That is, the DNA methylation levels in the PRC targeted genes are lower in CSC than in differentiated cancer cells, and also the bivalent chromatin structure may predominate in CSC. It is reasonable that the promoters of PRC targeted genes in CSC remain unmethylated to retain their gene expression plasticity in order to maintain their stem cell-like characteristics, because there is no DNA demethylase ever reported. Therefore, we attempted to compare the DNA methylation levels and chromatin structure of these PRC-targeted tumor suppressor genes (TSGs) between a cell population containing CSC and bulk tumor cells that consisted of differentiated cancer cells.

\section{Materials and methods}

Cell lines and cell culture. Two cancer cell lines were studied, the human breast cancer line MCF7 and the human liver cancer line Huh7. MCF7 and Huh7 cells were purchased from the American Type Culture Collection and the Japan Health Research Resources Bank (Osaka, Japan), respectively. The cells were cultured in DMEM, supplemented with 10\% FBS, 100 Units/ml penicillin G, and streptomycin (Gibco). Cells were maintained in $100-\mathrm{mm}$ culture dishes at $37^{\circ} \mathrm{C}$ in a $5 \% \mathrm{CO}_{2}$ atmosphere.

Methylation specific PCR (MSP) and quantitative RT-PCR. DNA was extracted using a DNeasy Tissue kit (Qiagen). Bisulfite treatment was performed using an EZ DNA Methylation Gold Kit (ZYMO Research) according to the manufacturer's protocol. MSP of the ten tumor suppressor genes was performed as previously described (27). The primer sequences used for MSP are listed in Table I. The PCR products were size-fractionated in $2 \%$ agarose gel and visualized by ethidium bromide staining. Total RNA was extracted using an RNeasy Mini kit (Qiagen). RNA was converted into cDNA using an Omniscript RT kit (Qiagen) according to the manufacturer's protocol. The primers used for quantitative RT-PCR were purchased from Applied Biosystems (ABI). Quantitative PCR was performed using an ABI Prism 7000 Sequence Detection system. GAPDH mRNA was used to normalize the variability in template loading.

Antibodies used for flow cytometry and chromatin immunoprecipitation (ChIP) assay. Monoclonal mouse anti-human CD24-fluorescein isothiocyanate (FITC) antibody (BD Pharmingen no. 555427) and a monoclonal mouse anti-CD44phycoerythrin (PE) antibody (BD Pharmingen no. 555479) were used for flow cytometry, while anti-H3K27me3 (Upstate no. 07-449), anti-H3K4me2 (Abcam no. ab11946), and antiEZH2 (Abcam no. ab3748) were used for ChIP assay.

Flow cytometry. A FACSVantage fluorescence-activated cell sorter (Becton-Dickinson) was used for our experiment. We isolated CD24-CD44+ cells from MCF7 as described previously (24). Side population cells from Huh7 were isolated as described previously (22). Huh7 cells were labeled with Hoechst 33342 dye either alone or in combination with verapamil.

Mammosphere formation assay. After cell sorting, 10,000 cells from each population were used for mammosphere formation assay. Mammosphere formation assay was performed as described previously (28). Mammospheres were grown in serum-free DMEM, supplemented with $20 \mathrm{ng} / \mathrm{ml}$ EGF and $20 \mathrm{ng} / \mathrm{ml} \mathrm{bFGF}$, for ten days. Pictures of the mammospheres were obtained using a Biozero (Keyence, Japan).

Bisulfite pyrosequencing. Bisulfite pyrosequencing was performed by Summit Pharmaceuticals International. The primer sequences used for PCR are as follows: 5'-GAGTTGTTTGA GGATTGGGATGT and 5'-TCATTTACCATTTTCCAAAC TTAC for RARb; and 5'-GTTTTTTAAGGGGTGTTGA and 5'-CRACCRCAAACTACTACC for SFRP1. 
Table II. The primers used for PCR in the study.

\begin{tabular}{|c|c|}
\hline Gene name & Sequence $\left(5^{\prime}-3^{\prime}\right)$ \\
\hline \multicolumn{2}{|l|}{ APC } \\
\hline Sense & GACTCGGAAATGGGGTAGGT \\
\hline Antisense & CCTGGATCCGAAGTACAGGA \\
\hline \multicolumn{2}{|l|}{ BRCA1 } \\
\hline Sense & TAAGCCGCAACTGGAAGAGT \\
\hline Antisense & CAGAAAGAGCCAAGCGTCTC \\
\hline \multicolumn{2}{|l|}{ CDH1 } \\
\hline Sense & AACAAAAGAACTCAGCCAAGTG \\
\hline Antisense & ACGCCACTGAGAGGGGGTGC \\
\hline \multicolumn{2}{|l|}{ DAPK } \\
\hline Sense & GAGAGGCTGCTTCGGAGTGTGAG \\
\hline Antisense & GCGGCGGGAACACAGCTAGG \\
\hline \multicolumn{2}{|l|}{ ESR1 } \\
\hline Sense & CCCTGACATTGGCTTAAACATCA \\
\hline Antisense & TCTTTGGGATCGCTCCAAAT \\
\hline \multicolumn{2}{|l|}{ FHIT } \\
\hline Sense & CTACCGTGGGGTCTTCTGG \\
\hline Antisense & TGTTGACAGCTGGGAATGAA \\
\hline \multicolumn{2}{|l|}{ MGMT } \\
\hline Sense & CCGGATATGCTGGGACAG \\
\hline Antisense & GACACTCACCAAGTCGCAAA \\
\hline \multicolumn{2}{|l|}{$\mathrm{RARb}$} \\
\hline Sense & GCCGAGAACGCGAGCGATCC \\
\hline Antisense & GGCCAATCCAGCCGGGGC \\
\hline \multicolumn{2}{|l|}{ RASSF1A } \\
\hline Sense & GTAAAGCTGGCCTCCAGAAA \\
\hline Antisense & GGAAGGAGCTGAGGAGAGC \\
\hline \multicolumn{2}{|l|}{ SFRP1 } \\
\hline Sense & AATTTCATGGGTTTGCAAGTATGA \\
\hline Antisense & TAAATAAAAGGGGGAGGAGGAAAG \\
\hline
\end{tabular}

Chromatin immunoprecipitation (ChIP) assays. ChIP assays were performed based on a modification of previously published methods $(19,29)$ using an Imprint ${ }^{\circledR}$ Chromatin Immunoprecipitation kit (Sigma-Aldrich). The primer sequences of PCR were designed to be within the $500 \mathrm{bp}$ region centering the transcriptional start sites (TSS) of each gene. The TSS of 10 TSGs were identified using DBTSS (http://dbtss.hgc.jp/). The precise primer sequences used for PCR are listed in Table II.

Quantification of PCR products in ChIP assays. Densitometry was performed using a LumiVision PRO 400EX (Aisin, Japan). The ratio of the intensity value of each PCR signal for dimethylated $\mathrm{H} 3 \mathrm{~K} 4$, trimethylated $\mathrm{H} 3 \mathrm{~K} 27$, and $\mathrm{EZH} 2$ to that of input DNA for ten tumor suppressor genes was calculated. The representative results of at least three independent experiments were averaged.
Statistical analysis. All statistical analyses were performed using SPSS Software, version 16.0.

\section{Results}

DNA methylation and the expression of TSGs in MCF7 cells. To examine the correlation between DNA methylation and the expression level of TSGs, we performed quantitative RT-PCR (Fig. 1A) and methylation specific PCR (MSP) (Fig. 1B) for 10 TSGs in MCF7 breast cancer cells. These 10 genes can also be targets of DNA methylation in various cancers $(6-8,19,30)$. Among them, we found 5 genes that were aberrantly methylated, and their mRNA expression levels were low. As previously reported, aberrant DNA hypermethylation was associated with repression of corresponding mRNA expression, with the exception of FHIT.

MCF7 cells have CSC enriched population. To enrich CSC, we selected MCF7 cells, a breast cancer cell line, because the existence of CSC in this cell line has been reported by several groups and the method to collect CSC appears to be well established (24,31). We sorted CD24-CD44+ cells as a population containing CSC from MCF7 cells by flow cytometry. Meanwhile, we sorted the major population, CD24+CD44 ${ }^{+}$ cells, as the population not containing CSC, i.e., the population of bulk tumor cells (Fig. 2A). We found the fraction of CD24-CD44+ were rare $(0.82 \%)$ in MCF7 cells. After cell sorting, we performed a sphere formation assay to confirm whether CD24-CD44+ cells from MCF7 cells contain CSC. It is known that breast cancer stem cells can form mammospheres in vitro, while differentiated breast cancer cells cannot $(28,32-33)$. We found CD24-CD44+ cells could form mammospheres in vitro (Fig. 2B). The number of mammospheres prepared from the population containing CSC was significantly greater than that of bulk tumor cells $(\mathrm{p}<0.01)$ (Fig. 2C). These results support that the cells we sorted from MCF7 CD24-CD44+ cells contained CSC.

DNA methylation level was lower in CSC. We compared the DNA methylation levels of the 5 aberrantly methylated genes, namely, APC, DAPK, RARb, RASSF1, and SFRP1, between these two populations in MCF7 cells by MSP. We found that unmethylated bands were seen in the population of CSC in DAPK and RARb genes, and more prominently in APC gene (Fig. 3A). On the other hand, methylated bands were prominently seen in the population of bulk tumor cells in RASSF1 and SFRP1.

To confirm and quantify these differences observed by MSP, we also performed bisulfite pyrosequencing to examine the DNA methylation levels of RARb (Fig. 3B) and SFRP1 (data not shown) in MCF7 cells. By using bisulfite pyrosequencing, we were able to evaluate the proportion of DNA methylation in each $\mathrm{CpG}$ island (34). The targeted region of the bisulfite pyrosequencing contained MSP primer sites and it also contained $11 \mathrm{CpG}$ islands in the RARb gene and 12 in the SFRP1 gene. We found that the DNA methylation level was lower in the population containing CSC than in the population of bulk tumor cells in both of the genes. Differences of approximately $4 \%$ in DNA methylation between these two 
A
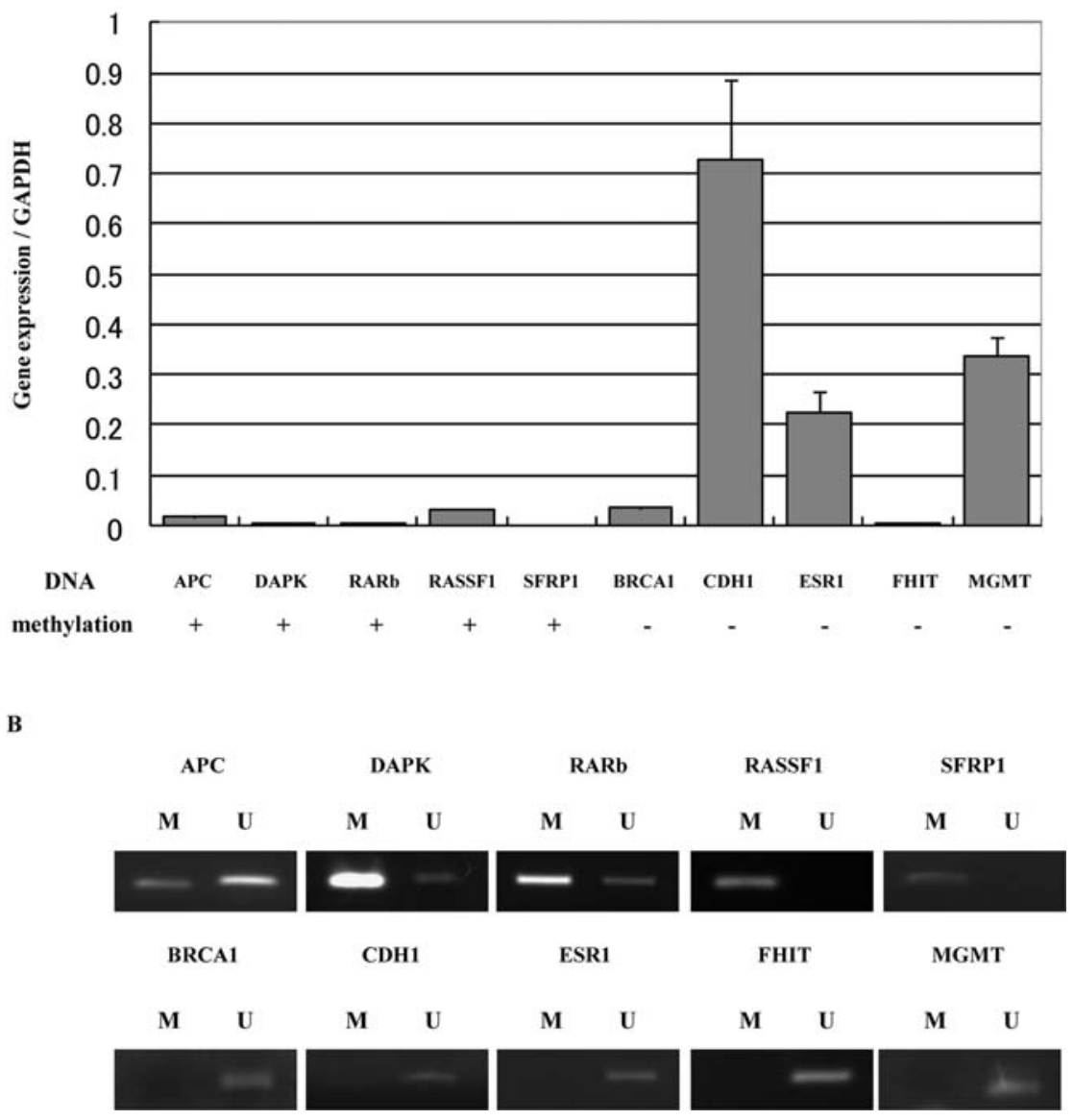

Figure 1. Gene expression and DNA methylation status of 10 TSGs in MCF7 cells. (A) Quantitative RT-PCR analysis of 10 TSGs. Mean expression levels of 10 TSGs are shown in a linear scale. Error bars, SEM of the averaged values. (B) MSP analysis of 10 TSGs. Five genes (APC, DAPK, RARB, RASSF1 and SFRP1) were aberrantly methylated.

A

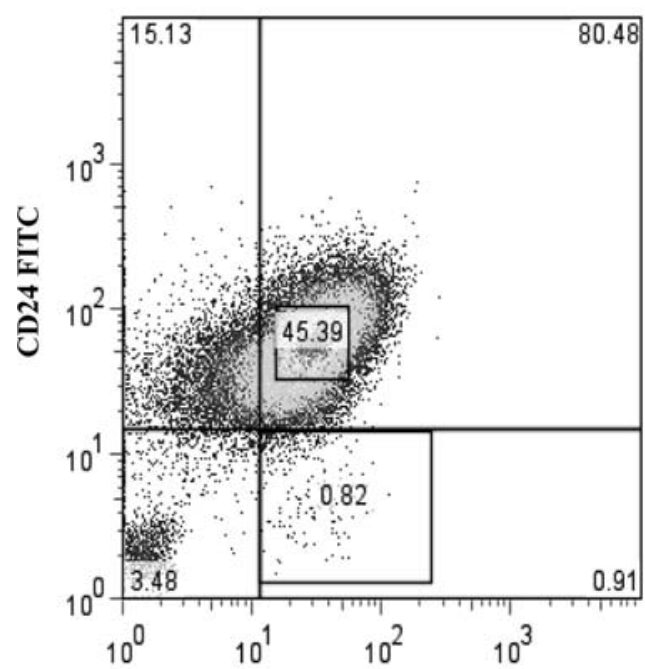

CD44 PE
B

C
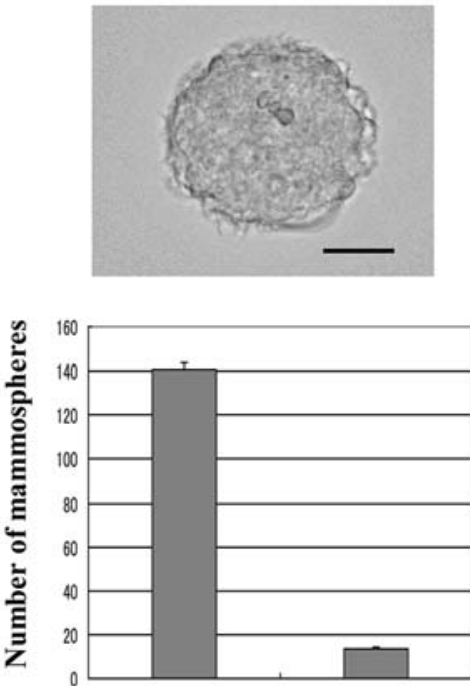

CSC

Bulk

Figure 2. (A) Analysis of flow cytometry for MCF7 cells. As a population containing CSC, we sorted CD24-CD44+ cells, and we sorted the major population, $\mathrm{CD} 24^{+} \mathrm{CD} 44^{+}$cells, as the population of bulk tumor cells. (B) Photograph of a mammosphere. Bar indicates $50 \mu \mathrm{m}$. (C) The number of mammospheres from two populations. The number of mammospheres prepared from the population containing CSC was significantly greater than that of bulk tumor cells $(\mathrm{p}<0.01)$. 
A

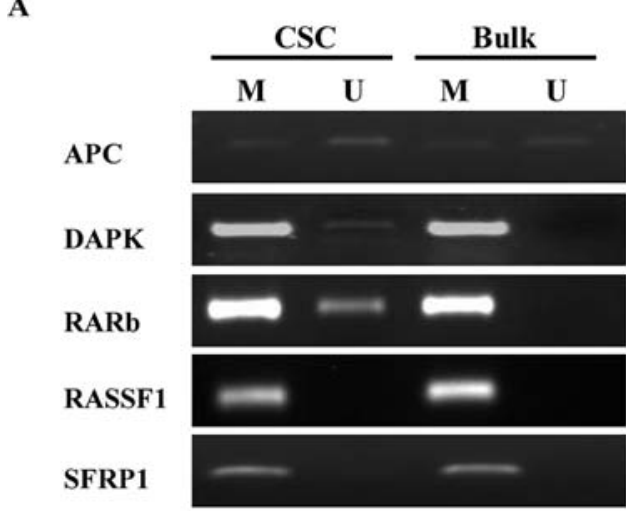

C
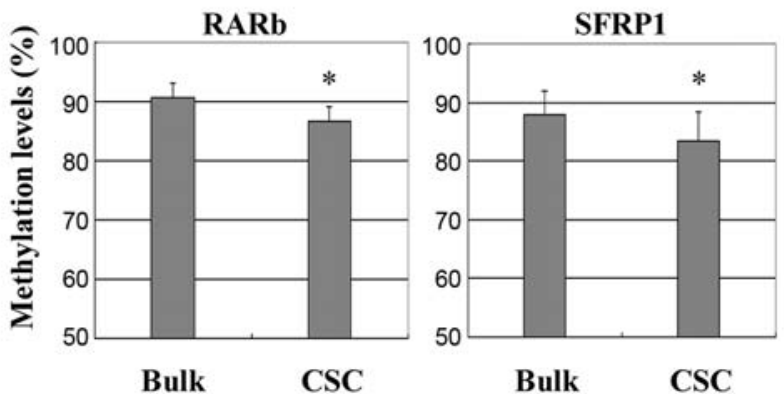

B

RARb Sequence 1

RARb Sequence 2

RARb Sequence 3

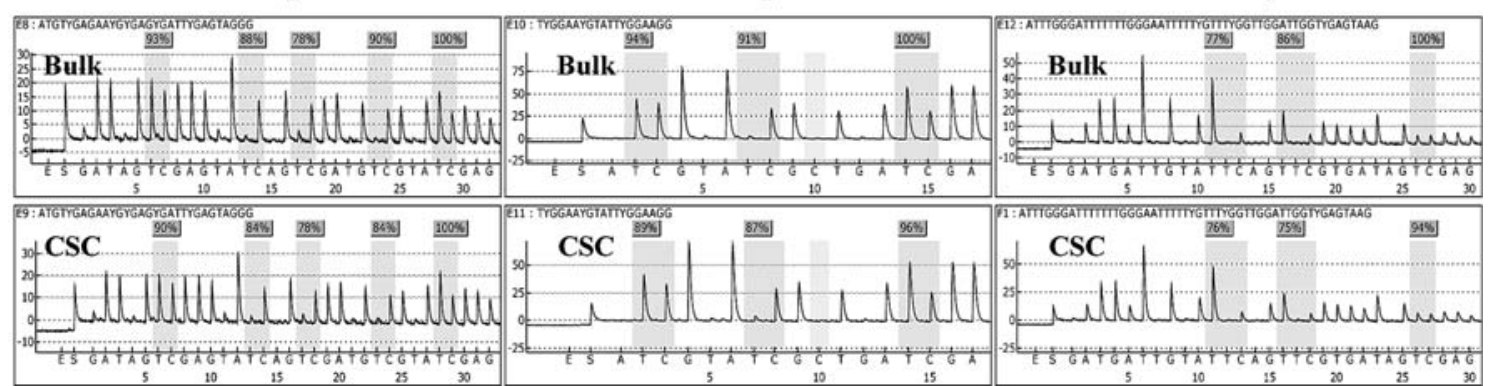

Figure 3. DNA methylation level was lower in CSC compared to bulk tumor cells. (A) MSP analysis of 5 TSGs. (B) Bisulfite pyrosequencing for RARb. The numbers indicate the DNA methylation level of each CpG island. (C) Mean DNA methylation levels. The difference in DNA methylation levels between these two populations was statistically significant ( $\mathrm{p}=0.002$ for $\mathrm{RARb}, \mathrm{p}=0.004$ for SFRP1).


Figure 4. (A) Analysis of flow cytometry for Huh7 cells. Left, as a population containing CSC, we sorted cells from the side population (SP) of Huh7 cells and sorted cells from the major population cells for the bulk tumor cells. Right, the SP cells disappeared with verapamil. (B) Results of MSP analysis of 9 TSGs. Three genes, namely CDKN2A, ESR1 and FHIT, are partially methylated. (C) The unmethylated bands were seen only in the SP cells for CDKN2A and ESR1 genes, and were prominent for FHIT gene. 
A

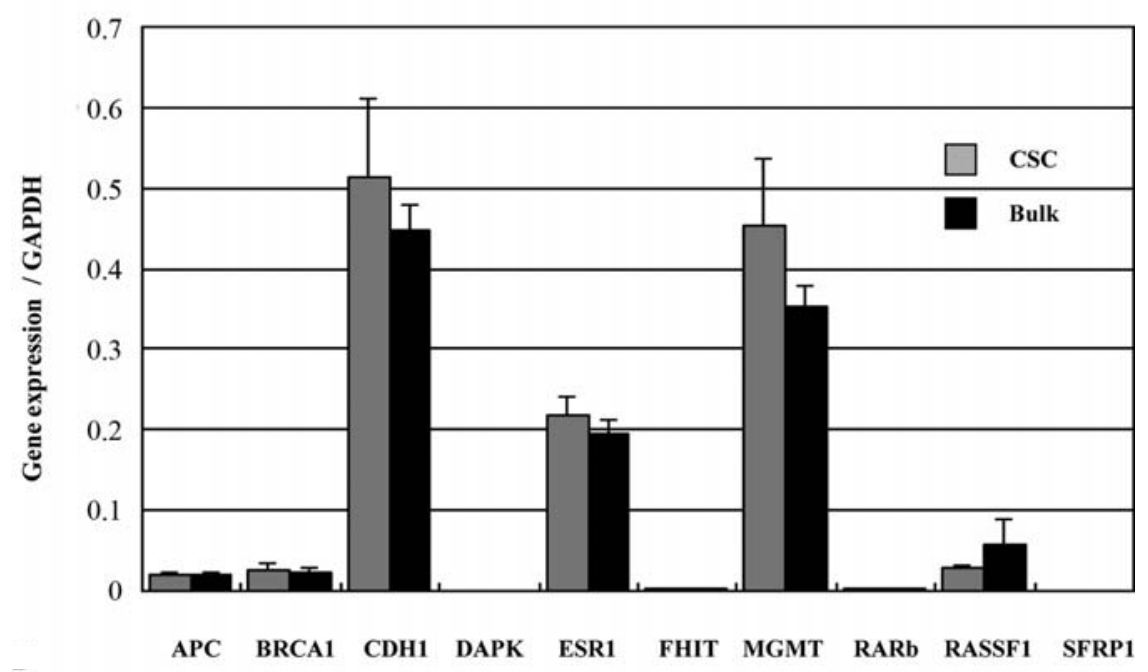

B

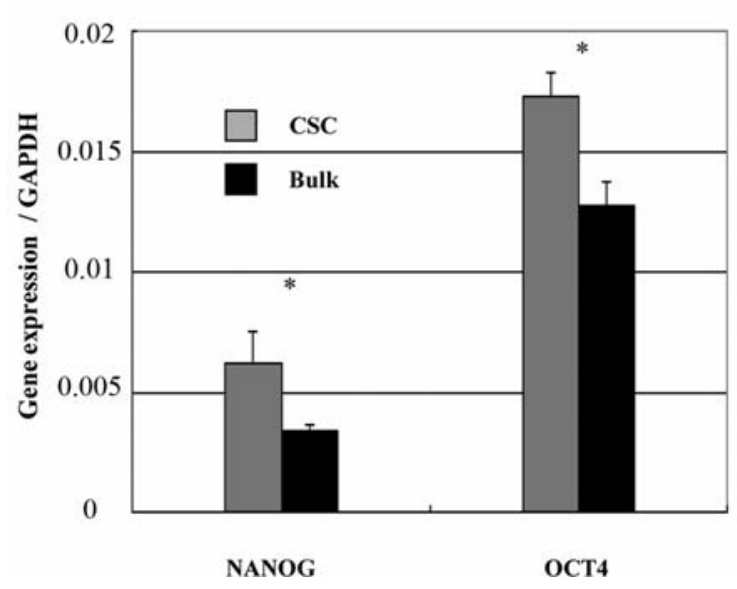

populations were observed in both genes and they were statistically significant ( $\mathrm{p}=0.002$ for $\mathrm{RARb}, \mathrm{p}=0.004$ for SFRP1) (Fig. 3C). These data strongly suggest that some of the promoters of PRC targeted genes in CSC remain unmethylated, while those in the majority of adult cancer cells are densely methylated. The difference in the DNA methylation level of about $4 \%$ indicates that the CSC are a minor, rare population even in populations containing CSC, as described elsewhere (33).

To determine if this epigenetic phenomenon is only restricted to MCF7 cells or is widely adapted to other CSC, we used another cell line, Huh7, from hepatocellular carcinoma. It is reported that side population cells of Huh7 cells harbor CSC-like properties (22). In this case, we sorted cells from the side population of Huh7 cells for the CSC containing population and sorted cells from the major population cells for the bulk tumor cells (Fig. 4A). Before cell sorting, we examined the DNA methylation status of the above PRC targeted gene promoters and found that CDKN2A, ESR1, and FHIT are partially methylated (Fig. 4B). After cell sorting, we compared the DNA methylation levels of these methylated genes between the CSC containing population and bulk tumor cell population. We found that unmethylated bands were only seen, or were more prominently seen in the
Figure 5. Comparison of the mRNA expression levels of TSGs, and two stemness genes, NANOG and OCT4, between CSC and bulk tumor cells. (A) The results of quantitative RT-PCR for 10 TSGs. Mean mRNA expression levels are shown in a linear scale. Error bars, SEM of the averaged values. (B) The results of quantitative RT-PCR for NANOG and OCT4. The mRNA expression levels were significantly higher in CSC for NANOG $(\mathrm{p}=0.036)$, and OCT4 $(\mathrm{p}=0.006)$.

population containing CSC compared to the bulk tumor cells in these genes (Fig. 4C).

Comparison of the expression level of TSGs, DNMTs, HMTs, and stemness genes between CSC and bulk tumor cells. We performed quantitative RT-PCR for the 10 TSGs mentioned above between CSC and bulk tumor cells from MCF7 in order to determine if any slight differences in DNA methylation led to differences in gene expression. However, we could not find any significant differences in gene expression (Fig. 5A). We also performed quantitative RT-PCR for DNA methyltransferases (DNMTs), histone methyltransferases (HMTs) (data not shown), and some stemness genes (Fig. 5B) to further characterize the CSC of MCF7 cells and to ascertain the reason for the differences in DNA methylation between CSC and bulk tumor cells. For DNMTs, we could not find any significant difference in expression between CSC and bulk tumor cells, although the expression of DNMT1 and DNMT3a was slightly lower in CSC. None of the HMTs genes showed any difference with respect to mRNA expression level between CSC and bulk tumor cells, while the expression levels of two stemness genes, namely, NANOG $(\mathrm{p}=0.036)$ and OCT4 $(\mathrm{P}=0.006)$, were higher in CSC (Fig. 5B). These two genes are transcriptional factors which are highly specific to ES cells, 
A

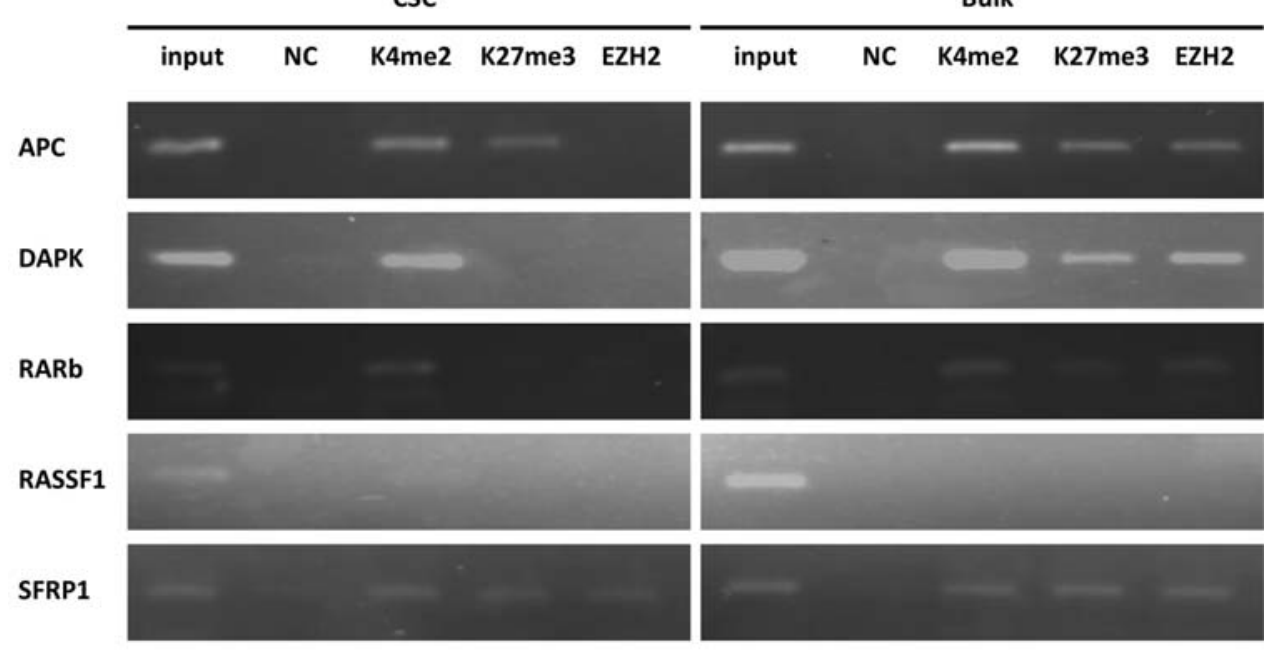

B

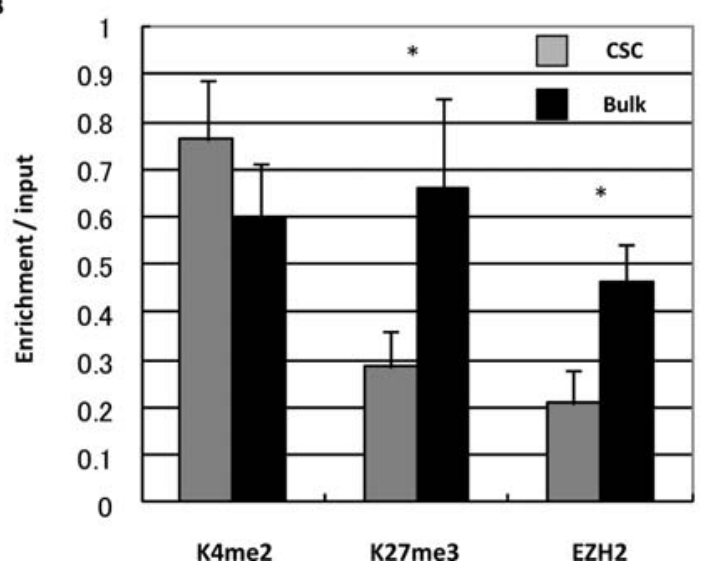

and play an essential role in maintaining the pluripotency of ES cells. It is also reported these genes are expressed in some cancer cells (35). These differences in expression of these genes further support the existence of CSC in MCF7 cells.

Unique and distinct chromatin patterns in CSC and bulk tumor cells. To further elucidate the epigenetic character of CSC and understand the mechanism of the differences in DNA methylation, we performed chromatin immunoprecipitation (ChIP) assay using antibodies to H3K4me2, H3K27me3, and EZH2 for MCF7 cells for 5 aberrantly methylated TSGs (Fig. 6A) and 5 unmethylated genes (data not shown). We found bivalent chromatin structures in both CSC and bulk tumor cells, but they were more prominent in bulk tumor cells, which was contrary to our hypothesis. Initially, we expected that only CSC possessed bivalent chromatin structures such as benign stem cells and bulk tumor cells lacking these structures by losing $\mathrm{H} 3 \mathrm{~K} 4 \mathrm{me} 2$, leading CSC to be relatively active in transcriptional state compared to bulk tumor cells. However, CSC were mainly marked by H3K4me 2 and the enrichment of H3K27me3 was significantly lower than bulk tumor cells. As a consequence, CSC remained in a relatively active transcriptional state compared to bulk tumor cells. The enrichment of H3K4me2 was not significantly different between CSC and bulk tumor cells, while there was a significant difference between that of H3K27me3 (p=0.048) and EZH2 (p=0.001) (Fig. 6B).
Figure 6. The results of ChIP assay between CSC and bulk tumor cells ChIP assay was performed using antibodies to H3K4me2 and H3K27me3, and EZH2 for MCF7 cells. (A) Photographs of ChIP PCR for 5 aberrantly methylated TSGs for CSC and bulk tumor cells of MCF7 cells. A negative control with antibodies against normal mouse IgG is included for comparison. We found bivalent chromatin structures in both CSC and bulk tumor cells, but they were more prominent in bulk tumor cells. (B) Mean enrichment levels of $\mathrm{H} 3 \mathrm{~K} 4 \mathrm{me} 2$ and $\mathrm{H} 3 \mathrm{~K} 27 \mathrm{me} 3$, and EZH2 for 10 TSG. The enrichment of $\mathrm{H} 3 \mathrm{~K} 4 \mathrm{me} 2$ was not significantly different between CSC and bulk tumor cells, however, those of H3K27me3 (p=0.048) and EZH2 ( $\mathrm{p}=0.001)$ were significantly different. Error bars indicate SEM.

\section{Discussion}

DNA methylation is closely related to the differentiation of ES cells (11-13). In differentiation, the changes of DNA methylation are notably found on the promoters of developmentally regulated genes, such as the transcription factors OCT4 and $N A N O G$ genes, which are expressed in pluripotent ES cells. For example, sequential DNA methylation of these genes occurs in human NT2 cells during neural differentiation (13). Vice versa dedifferentiation of fibroblasts to iPS cells results in a loss of DNA hypermethylation (36). It has been reported that bivalent chromatin structures premark the tumor suppressor genes that include targets of polycomb group proteins for aberrant DNA hypermethylation (19). Polycomb group proteins are epigenetic gene silencers, and play an essential role in the maintenance of embryonic and adult stem cells (37-42). These genes are kept in a low state of DNA methylation in undifferentiated ES cells and embryonic cancer cells, while they are densely methylated in relatively differentiated adult cancer cells. The bivalent chromatin structure exists in ES cells and embryonal cancer cells, and if some differentiation cues are given to these cells, DNA hypermethylation occurs (19).

In the present study, we found epigenetic regulation of CSC was in some ways very similar to these undifferentiated cells, while it was different in other ways. The DNA methylation pattern was the same in CSC as in these undifferentiated cells. However, the chromatin pattern in CSC was different 
from that in undifferentiated cells. We observed a significant difference in epigenetics between CSC and bulk tumor cells, however, there was no difference in mRNA expression of targeted genes. There are two possible explanations for this. One is that although differences in gene expression exist between less methylated CSC and densely methylated bulk tumor cells, the differences in gene expression levels were too small to be detected by quantitative RT-PCR as the true CSC were quite rare population estimated to be less than $5 \%$ even in CSC-containing population (33). Another possibility is that there is no difference in gene expression between less methylated CSC and densely methylated bulk tumor cells, however, CSC are in a state of readiness for transcription until certain cues occur, although we were unable to identify these cues in the present study.

It is noteworthy that the bivalent chromatin structure seen in benign stem cells was not specific to CSC, but was observed ubiquitously in cancer cells. We found a distinct difference in chromatin structure between CSC and bulk tumor cells. These findings suggest the differentiation of cancer cells is also controlled by epigenetic mechanisms, as is the case in organ systems.

Concerning gene expression, the balance between active chromatin mark and repressive chromatin mark moves towards a more active transcriptional state in CSC compared to bulk tumor cells, because the transcriptionally repressive $\mathrm{H} 3 \mathrm{~K} 27 \mathrm{me} 3$ marks are found less often in CSC. The shift towards a transcriptionally active epigenetic state in CSC is in concordance with the patterns of DNA methylation that we have found. The enrichment of EZH2 in these 10 TSGs is significantly higher in bulk tumor cells than in CSC, which was also the case for H3K27me3. The coincidence between DNA methylation and chromatin modification in bulk tumor cells might indicate that they achieved repressive mark of H3K27me3 or EZH2 during differentiation.

CSC is thought to be a cause of cancer relapse, and to eradicate cancer we have to eradicate CSC. In order to achieve this, more details of the characteristics of CSC need to be elucidated. The high expression of two transcriptional factors, NANOG and OCT4, and a less methylated state of DNA in CSC may raise the possibility that CSC can be reprogrammed to other differentiated tissues. In turn, differentiation therapy may become a novel therapeutic strategy for cancer treatment in the future.

We found that CSC possess unique epigenetic states, that is, significantly less DNA methylation and significantly less repressive chromatin marks. Thereby, CSC were kept in relatively active transcriptional state compared to bulk tumor cells. Although many features of CSC have been reported, no report has directly demonstrated any differences in epigenetics in CSC, which is thought to be one of the essential characteristics of stem cells. Further characterization of CSC on epigenetic regulations, should allow us to identify bona fide targets that define tumor stemness, and facilitate the development of targeted therapies to eradicate human malignancies.

\section{Acknowledgements}

We wish to thank Ms. M. Shibuya and Ms. M. Yamamoto for their excellent technical assistance with the molecular analyses.
This study was supported in part by Grants-in-Aid for Scientific Research on Priority Areas from the Ministry of Education, Culture, Sports, Science, and Technology of Japan to KS. The authors declare that they have no competing interests.

\section{References}

1. Jones PA and Baylin SB: The fundamental role of epigenetic events in cancer. Nat Rev Genet 3: 415-428, 2002.

2. Jones PA and Laird PW: Cancer epigenetics comes of age. Nat Genet 21: 163-167, 1999.

3. Feinberg AP and Tycko B: The history of cancer epigenetics. Nat Rev Cancer 4: 143-153, 2004.

4. Jones PA and Baylin SB: The epigenomics of cancer. Cell 128: 683-692, 2007.

5. Issa JP: Aging, DNA methylation and cancer. Crit Rev Oncol Hematol 32: 31-43, 1999.

6. Baylin SB and Ohm JE: Epigenetic gene silencing in cancer - a mechanism for early oncogenic pathway addiction? Nat Rev Cancer 6: 107-116, 2006.

7. Esteller M: Cancer epigenetics: DNA methylation and chromatin alterations in human cancer. Adv Exp Med Biol 532: 39-49, 2003.

8. Esteller M: Epigenetics in cancer. N Engl J Med 358: 1148-1159, 2008.

9. Razin A and Cedar H: DNA methylation and gene expression. Microbiol Rev 55: 451-458, 1991.

10. Herman JG and Baylin SB: Gene silencing in cancer in association with promoter hypermethylation. N Engl J Med 349: 2042-2054, 2003

11. Fouse SD, Shen Y, Pellegrini M, et al: Promoter CpG methylation contributes to ES cell gene regulation in parallel with Oct4/ Nanog, PcG complex, and histone H3 K4/K27 trimethylation. Cell Stem Cell 2: 160-169, 2008.

12. Jackson M, Krassowska A, Gilbert N, et al: Severe global DNA hypomethylation blocks differentiation and induces histone hyperacetylation in embryonic stem cells. Mol Cell Biol 24: 8862-8871, 2004.

13. Deb-Rinker P, Ly D, Jezierski A, Sikorska M and Walker PR: Sequential DNA methylation of the Nanog and Oct-4 upstream regions in human NT2 cells during neuronal differentiation. J Biol Chem 280: 6257-6260, 2005.

14. Kouzarides T: Chromatin modifications and their function. Cell 128: 693-705, 2007.

15. Bernstein BE, Mikkelsen TS, Xie X, et al: A bivalent chromatin structure marks key developmental genes in embryonic stem cells. Cell 125: 315-326, 2006.

16. Lee TI, Jenner RG, Boyer LA, et al: Control of developmental regulators by Polycomb in human embryonic stem cells. Cell 125: 301-313, 2006.

17. Boyer LA, Plath K, Zeitlinger J, et al: Polycomb complexes repress developmental regulators in murine embryonic stem cells. Nature 441: 349-353, 2006.

18. Holden C: Development. Gene-suppressing proteins reveal secrets of stem cells. Science 312: 349, 2006.

19. Ohm JE, McGarvey KM, Yu X, et al: A stem cell-like chromatin pattern may predispose tumor suppressor genes to DNA hypermethylation and heritable silencing. Nat Genet 39: 237-242, 2007.

20. Al-Hajj M, Wicha MS, Benito-Hernandez A, Morrison SJ and Clarke MF: Prospective identification of tumorigenic breast cancer cells. Proc Natl Acad Sci USA 100: 3983-3988, 2003.

21. Bonnet D and Dick JE: Human acute myeloid leukemia is organized as a hierarchy that originates from a primitive hematopoietic cell. Nat Med 3: 730-737, 1997.

22. Chiba T, Kita K, Zheng YW, et al: Side population purified from hepatocellular carcinoma cells harbors cancer stem celllike properties. Hepatology 44: 240-251, 2006.

23. O'Brien CA, Pollett A, Gallinger S and Dick JE: A human colon cancer cell capable of initiating tumour growth in immunodeficient mice. Nature 445: 106-110, 2007.

24. Ponti D, Costa A, Zaffaroni N, et al: Isolation and in vitro propagation of tumorigenic breast cancer cells with stem/ progenitor cell properties. Cancer Res 65: 5506-5511, 2005.

25. Ricci-Vitiani L, Lombardi DG, Pilozzi E, et al: Identification and expansion of human colon-cancer-initiating cells. Nature 445: 111-115, 2007

26. Singh SK, Hawkins C, Clarke ID, et al: Identification of human brain tumour initiating cells. Nature 432: 396-401, 2004. 
27. Herman JG, Graff JR, Myohanen S, Nelkin BD and Baylin SB: Methylation-specific PCR: a novel PCR assay for methylation status of CpG islands. Proc Natl Acad Sci USA 93: 9821-9826, 1996.

28. Dontu G, Abdallah WM, Foley JM, et al: In vitro propagation and transcriptional profiling of human mammary stem/ progenitor cells. Genes Dev 17: 1253-1270, 2003.

29. Horak CE, Mahajan MC, Luscombe NM, Gerstein M, Weissman SM and Snyder M: GATA-1 binding sites mapped in the beta-globin locus by using mammalian chIp-chip analysis. Proc Natl Acad Sci USA 99: 2924-2929, 2002.

30. Toyota M and Issa JP: Epigenetic changes in solid and hematopoietic tumors. Semin Oncol 32: 521-530, 2005.

31. Phillips TM, McBride WH and Pajonk F: The response of CD24(-/low)/CD44+ breast cancer-initiating cells to radiation. J Natl Cancer Inst 98: 1777-1785, 2006.

32. Liao MJ, Zhang CC, Zhou B, et al: Enrichment of a population of mammary gland cells that form mammospheres and have in vivo repopulating activity. Cancer Res 67: 8131-8138, 2007.

33. Mani SA, Guo W, Liao MJ, et al: The epithelial-mesenchymal transition generates cells with properties of stem cells. Cell 133: 704-715, 2008

34. Colella S, Shen L, Baggerly KA, Issa JP and Krahe R: Sensitive and quantitative universal Pyrosequencing methylation analysis of CpG sites. Biotechniques 35: 146-150, 2003.
35. Ezeh UI, Turek PJ, Reijo RA and Clark AT: Human embryonic stem cell genes OCT4, NANOG, STELLAR, and GDF3 are expressed in both seminoma and breast carcinoma. Cancer 104: 2255-2265, 2005

36. Takahashi K, Tanabe K, Ohnuki M, et al: Induction of pluripotent stem cells from adult human fibroblasts by defined factors. Cell 131: 861-872, 2007.

37. Sparmann A and van Lohuizen M: Polycomb silencers control cell fate, development and cancer. Nat Rev Cancer 6: 846-856, 2006.

38. Bracken AP, Dietrich N, Pasini D, Hansen KH and Helin K: Genome-wide mapping of Polycomb target genes unravels their roles in cell fate transitions. Genes Dev 20: 1123-1136, 2006.

39. Kennison JA: The Polycomb and trithorax group proteins of Drosophila: trans-regulators of homeotic gene function. Annu Rev Genet 29: 289-303, 1995.

40. Levine SS, King IF and Kingston RE: Division of labor in polycomb group repression. Trends Biochem Sci 29: 478-485, 2004.

41. Lund $\mathrm{AH}$ and van Lohuizen $\mathrm{M}$ : Polycomb complexes and silencing mechanisms. Curr Opin Cell Biol 16: 239-246, 2004.

42. Pirrotta V: Polycomb silencing mechanisms and genomic programming. Ernst Schering Res Found Workshop, pp97-113, 2006. 